\title{
Energética de Complexos Metálicos
} Algumas perspectivas

\author{
MARIA DAS DORES M. C. RIBEIRO DA SILVA*
}

\section{Objectivos da Termoquímica Molecular}

A Termoquímica Molecular tem como objectivo fundamental o estudo da estabilidade das moléculas. Falar de estabilidade de uma molécula, existente em determinadas condições físicas e numa vizinhança química particular, pode corresponder a alguma ambiguidade, uma vez que se impõe explicitar relativamente a quê tal conceito se define.

A energia de Gibbs padrão de reacção pode ser encarada como uma medida da estabilidade termodinâmica relativa de moléculas que participam em reacções químicas semelhantes, a pressão e temperatura fixas. Tal é consequência de a constante de equilibrio para uma dada reacção, K , a uma determinada temperatura e à pressão padrão, se relacionar com a energia de Gibbs padrão de reacção, $\Delta_{r} G_{m}^{0}$, por (1).

$$
\Delta_{\mathrm{r}} G_{\mathrm{m}}^{0}=-R T \ln \mathrm{K}
$$

Por sua vez, a energia de Gibbs padrão da reacção pode ser calculada, a uma dada temperatura de referência, pela bem conhecida equação (2), a partir do conhecimento dos valores da entalpia padrão da reacção, $\Delta_{\mathrm{r}} H_{\mathrm{m}}^{0}$, e da entropia padrão de reacção, $\Delta_{\mathrm{r}} S_{\mathrm{m}}^{0}$, à mesma temperatura, $T$.

$$
\Delta_{\mathrm{r}} G_{\mathrm{m}}^{0}=\Delta_{\mathrm{r}} H_{\mathrm{m}^{-}}^{0}-T \Delta_{\mathrm{r}} S_{\mathrm{m}}^{0}
$$

A temperatura ambiente, em particular para reacções em fase gasosa, o termo $T \Delta_{\mathrm{r}} S_{\mathrm{m}}^{0}$ é frequentemente pequeno quando comparado com $\Delta_{\mathrm{r}} H_{\mathrm{m}}^{0}$, implicando que o termo entalpia de reacção seja o termo dominante na equação (2). $\mathrm{O}$ valor de $\Delta_{\mathrm{r}} H_{\mathrm{m}}^{\mathrm{O}}$ é calculado a partir das entalpias de formação padrão dos reagentes e produtos envolvidos na reacção, pelo que estes parâmetros podem corresponder a potenciais indicadores de estabilidade termodinâmica.

0 conhecimento de parâmetros energéticos das moléculas, sendo útil em diversas áreas de ciência e tecnologia, tem primordial importância na contribuição para o estudo de relações de interdependência entre energética, estrutura e reactividade. A interpretação de parâmetros termoquímicos, nomeadamente de valores de entalpias de formação, pode conduzir a uma melhor compreensão da natureza das ligações químicas, bem como ao conhecimento e/ou confirmaçäo da energética de tais ligações e, consequentemente, servir de suporte às correlaçōes entre características estruturais e reaccionais dos compostos de que essas ligações fazem parte. É, assim, de salientar que os estudos termoquímicos não estão sempre directamente ligados com a estabilidade termodinâmica de moléculas como um todo, sendo frequente relacionarem-se com a estabilidade termodinâmica das ligações químicas características das moléculas objecto de interesse. De facto, o conhecimento da energia molecular pode ser obtido directamente por via experimental ou por análise de resultados relativos a unidades estruturais simples, com a consequente extrapolação para compostos mais complexos, de que aquelas unidades são parte integrante. Há uma vasta evidência experimental para suporte de princípios de aditividade em compostos envolvendo elementos do segundo período $(n=2)$ e, em parte, do terceiro $(n=3 ; \mid=0,1)$. Tal não acontece com elementos das séries de transição, para os quais os esquemas de transferibilidade de energias de grupos de átomos não são facilmente aplicáveis, fundamentalmente por falta de dados chave de entalpias de formação dos respectivos compostos.

O estudo termoquímico de complexos de metais de transição tem constituído um desafio pelo qual o grupo de Termoquímica da Faculdade de Ciências do Porto se tem vindo a interessar, não só pela contribuição para o conhecimento desses parâmetros em si, mas também por a partir deles se poderem estabelecer esquemas de previsão de razoável fiabilidade. Em particular, tem sido dada especial atenção ao estudo de complexos de metais de transição com diferentes ligandos, envolvendo ligações metaloxigénio, metal-azoto e metal-enxofre. É, assim, de referir estudos de complexos metálicos com ligandos bidentados $(\mathrm{HL})$ da família das $\beta$-dicetonas ${ }^{[1,2]}$ (ligações metal-oxigénio), da familia das dimetilglióximas $^{[3]}$ (ligações metal-azoto), da família dos ditiocarbamatos ${ }^{\mid 4,71}$ (ligações metal-enxofre), das famílias dos aminoácidos $^{[8,9]}$ e das hidroxiquinoli-

\footnotetext{
* Centro de Investigaçāo em Química, Departamento de Química, Faculdade de Ciências, Universidade do Porto, Rua do Campo Alegre, 687. P-4169-007 Porto, Portugal
} 
nas ${ }^{[10 !}$ (ligaçōes metal-oxigénio e metalazoto), das familias das $\beta$-tiodicetonas $^{[11]}$ e das acilcalcogenoureias ${ }^{[12,13]}(\mid \mathrm{i}$ gações metal-oxigénio e metal-enxofre) e estudos de complexos metálicos com ligandos tetradentados $\left(\mathrm{H}_{2} \mathrm{~L}\right)$ da familia das bases de Schifff ${ }^{14]}$ (ligações metaloxigénio e metal-azoto).

Um aspecto importante da termoquímica de complexos de coordenação relaciona-se com a energética de ligação metal-ligando que pode ser considerada no contexto de esquemas de energias de ligação ou no de energias de dissociação. Estas últimas, não traduzindo uma medida directa das energias de ligação devido à reorganização dos radicais ligandos, são de primordial importância em termos de reactividade, podendo adequar-se à discussão da energética relativa dessas ligações. A interpretação de parâmetros termoquímicos, nomeadamente de valores de entalpias de formação, beneficia, assim, de uma boa compreensão da natureza das ligações químicas, sendo o inverso igualmente verdade.

A entalpia média de dissociação homolítica metal-ligando em complexos de metais de transição $\mathrm{ML}_{n}$ é definida como $<D H>(M-L)=\Delta H / n$, em que $\Delta H$ é a entalpia do processo hipotético descrito pela equação química (3).

$$
\mathrm{ML}_{n}(\mathrm{~g}) \rightarrow \mathrm{M}(\mathrm{g})+\mathrm{nL}(\mathrm{g})
$$

O valor de $\Delta H$ é calculável a partir dos valores das entalpias de formação de todas as espécies envolvidas no processo, o que implica a necessidade da frequente determinação experimental dos valores desconhecidos das entalpias de formação dos complexos e ligandos, nas fases condensada e gasosa.

\section{Metodologia de trabalho}

A energia de dissociação de uma ligação química $\mathrm{M}-\mathrm{L}$ na molécula $\mathrm{ML}_{n}, D_{0}^{0}$, define-se como $\Delta_{\mathrm{r}} U_{\mathrm{m}}^{0}$, a $T=0 \mathrm{~K}$, para a reacção descrita por (4), estando todas as espécies envolvidas na reacção nos respectivos estados vibracionais fundamentais.

$M L_{n}(g) \rightarrow M L_{n-1}(g)+L(g)$

Por outro lado, define-se entalpia de dissociação $\mathrm{DH}^{0}{ }_{298.15}$ K dessa mesma ligação química como a variação de entalpia, $\Delta r H_{m}^{0}$, a $T=298.15 \mathrm{~K}$, para a referida reacção. A diferença entre $D_{0}^{0} \mathrm{e}$ $\mathrm{DH}_{298.15 \mathrm{~K}} \mathrm{e}$ usualmente pequena e a diferença máxima é de $10 \mathrm{~kJ} \mathrm{~mol}^{-1}$ para a dissociação de uma molécula poliatómica, se nenhum dos graus de liberdade vibracional perdido na dissociação fosse excitado, a $T=298.15 \mathrm{~K}$. Ao longo deste texto, para uma maior facilidade de escrita, a entalpia de dissociação da

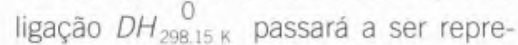
sentada na forma simplificada $\mathrm{DH}$.

Se todas as ligações metal-ligando forem rompidas e consideradas como equivalentes, de acordo com a equação química traduzida em (3), o valor da entalpia de reacção é calculável pela equação (5), sendo $\Delta_{\mathrm{r}} H_{\mathrm{m}}^{0} / \mathrm{n}=\langle\mathrm{DH}>(\mathrm{M}$ L), a entalpia média de dissociação da ligação (M-L).

$$
\begin{aligned}
\Delta_{\mathrm{r}} H_{\mathrm{m}}^{0}= & \Delta_{\mathrm{f}} H_{\mathrm{m}}^{0}(\mathrm{M}, \mathrm{g})+\mathrm{n} \Delta_{\mathrm{f}} H_{\mathrm{m}}^{0}(\mathrm{~L}, \mathrm{~g}) \\
& -\Delta_{\mathrm{f}} H_{\mathrm{m}}^{\mathrm{O}}\left(\mathrm{ML} \mathrm{L}_{\mathrm{n}, \mathrm{g})}\right.
\end{aligned}
$$

A entalpia de dissociação, incluindo a energia de reorganização do radical (energia associada com a diferença de estrutura do ligando coordenado e livre), não traduz directamente o valor da força da ligação química. Para um ligando do tipo HL, que forma um complexo metálico $\mathrm{ML}_{n}$ o efeito da energia de reorganização pode, numa primeira aproximação, ser atenuado considerando a entalpia de dissociação da ligação metal-ligando relativamente à entalpia de dissociação da ligação ao hidrogénio no ligando, isto é, $\langle D H>(\mathrm{M}-\mathrm{L})-D H(\mathrm{H}$ L). Assim, pode concluir-se que a diferença $\angle D H\rangle(\mathrm{M}-\mathrm{L})-\mathrm{DH}(\mathrm{H}-\mathrm{L})$ é adequada para analisar valores de energias de dissociação relativos, revestindo-se tal análise de particular relevância nos casos em que o valor de $D H(H-L)$ é desconhecido.
Considerando que $D H(\mathrm{H}-\mathrm{L})$ corresponde ao valor $\Delta_{\mathrm{r}} H_{\mathrm{m}}^{0}$ para a reacção traduzida pela equação química (6), a combinação deste parâmetro com o referente $a<D H>(M-L)$, obtido a partir de (3) e (5), permite estabelecer a equação (7), a partir da qual se calculam o valores de $\langle D H\rangle(\mathrm{M}-\mathrm{L})-D H(\mathrm{H}-\mathrm{L})$, usando resultados de medições experimentais.

$\mathrm{HL}(\mathrm{g}) \rightarrow \mathrm{H}(\mathrm{g})+\mathrm{L}(\mathrm{g})$

$<D H>(\mathrm{M}-\mathrm{L})-D H(\mathrm{H}-\mathrm{L})=$

$=\frac{1}{n}\left\{\Delta_{\mathrm{f}} H_{m}^{0}(M, g)-\Delta_{\mathrm{f}} H_{m}^{0}\left(M L_{n}, g\right)\right\}+$

$+\Delta_{f} H_{m}^{0}(H L, g)-\Delta_{f} H_{m}^{0}(H, g)$

$O$ cálculo do valor de $\langle D H>(M-L)$ implica que se conheça o valor de $D H(H-L)$.

\section{Técnicas experimentais}

A necessidade de eliminar os efeitos energéticos das interacçōes intermoleculares, existentes em fases condensadas, impõe que o estudo energético de uma dada molécula se faça no estado gasoso, no qual se pode admitir a inexistência de tal tipo de interacções. Uma revisão dos principais métodos experimentais usados no estudo da estabilidade de espécies químicas foi publicada recentemente por Martinho Simões, ${ }^{[15]}$ que apresenta de forma concisa uma panorâmica geral sobre o assunto. A determinação experimental directa de parâmetros termodinâmicos para moléculas em fase gasosa nem sempre é viável, pelo que a medição de propriedades energéticas dessas moléculas, em fase condensada, e das correspondentes propriedades relativas a processos de transição de fase pode ser de grande interesse. De facto, o conhecimento de valores de entalpias de formação das moléculas, em fase condensada, e de valores de entalpias de sublimação/va- 
porização dessas moléculas permite, por conjugação dos mesmos, calcular os valores das correspondentes entalpias de formação em fase gasosa, constituindo uma alternativa à obtenção, por um método directo, de tais parâmetros.

A principal técnica experimental mais usada no estudo dos complexos metálicos no estado cristalino é a calorimetria isoperibol de solução - reacção (RSC reaction/solution calorimetry), que foi reconhecida como um bom método para investigar a estabilidade termodinâmica destes compostos, tendo vindo a ser usada para a análise sistemática das entalpias de dissociação de ligações metal-ligando em compostos de metais de transição. $\mathrm{O}$ recurso à técnica de calorimetria de combustão (CC - combustion calorimetry), quer em bomba estática (SBC - static bomb calorimetry) quer em rotativa ( $\mathrm{RBC}$ - rotating bomb calorimetry), é necessário para obter informação acerca de parâmetros energéticos relativos a ligandos em fase condensada. Os parâmetros energéticos em fase gasosa, para complexos e ligandos, são obtidos conjugando os valores da fase condensada com os de entalpias de evaporação, obtidos calorimetricamente (CM - Calvet Microcalorimetry) ou por efusão de Knudsen (KE - Knudsen-effusion). 0 diagrama da figura 1 apresenta de forma esquenática a sequência de determinações experimentais para se calcular o valor de $\langle D H\rangle(\mathrm{M}-\mathrm{L})-D H(\mathrm{H}$ L).

\section{Calorimetria de solução-reacção}

A calorimetria de solução-reacção permite determinar a entalpia de formação de substâncias, de forma indirecta, a partir da medição de calores de solução ou de reacção num solvente apropriado. Os valores entálpicos são obtidos convertendo, a partir dos equivalentes energéticos do sistema, variações de temperatura medidas em sucessivas reacções num dado solvente, de acordo com o esquema apresentado na figura 2.

\section{Calorimetria de combustão}

A calorimetria de combustão é a técnica mais usada para a determinação da entalpia de formação de compostos orgâ-

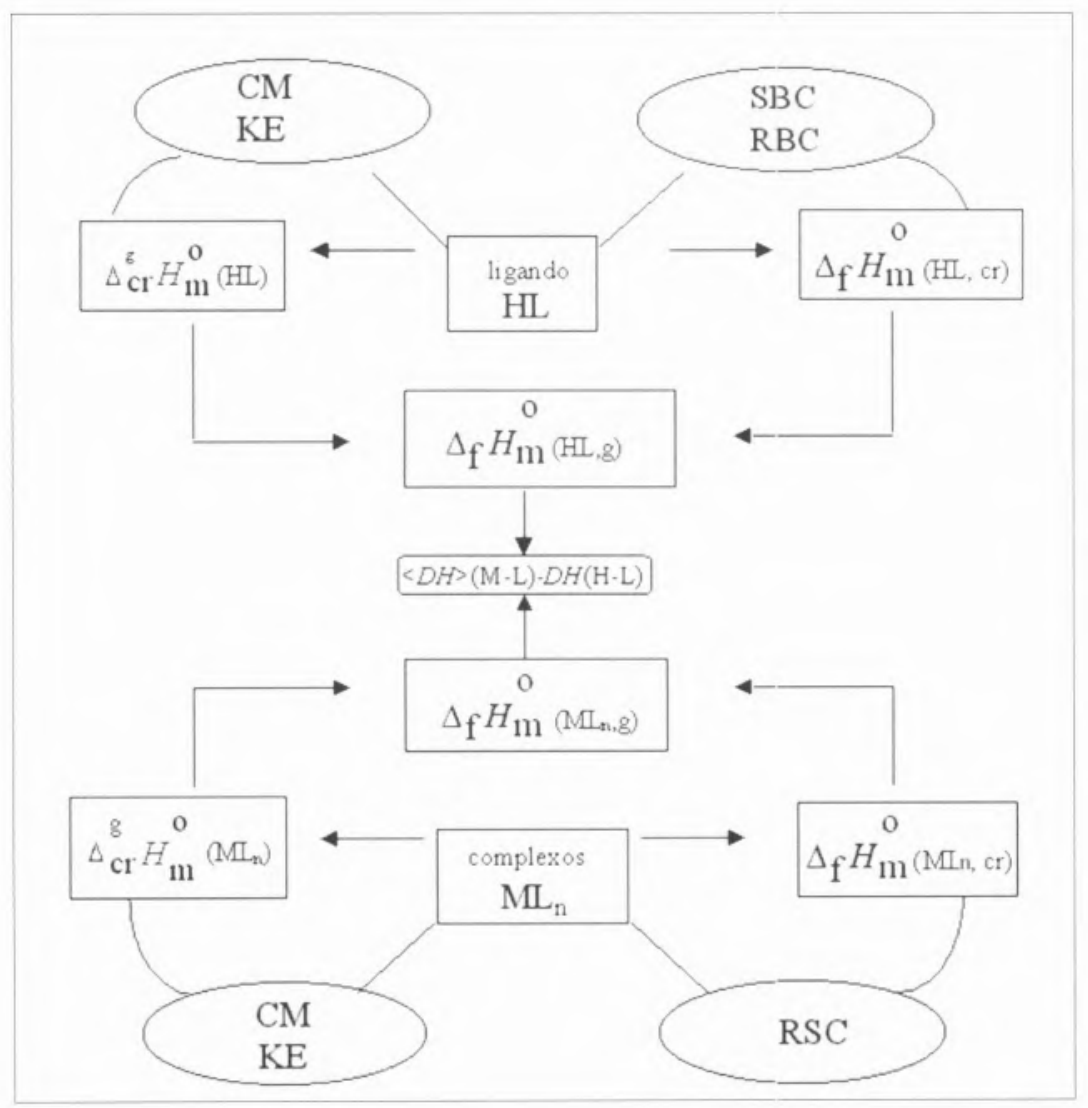

figura 1 - Sequência de determinaçōes experimentais para o estudo da energética de complexos metálicos

nicos. A reacção deve ser completa, pelo que é levada a cabo na presença de um grande excesso de oxigénio. A variante de bomba estática é aplicada a compostos contendo carbono, oxigénio, azoto e hidrogénio, cuja reacção é traduzida pela equação química (8).

$$
\begin{aligned}
& \mathrm{C}_{\mathrm{a}} \mathrm{H}_{\mathrm{b}} \mathrm{O}_{\mathrm{c}} \mathrm{N}_{\mathrm{d}}(\mathrm{cr}, \mathrm{I})+(\mathrm{a}+\mathrm{b} / 4-\mathrm{c} / 2) \mathrm{O}_{2}(\mathrm{~g}) \\
& \rightarrow \mathrm{a} \mathrm{CO}_{2}(\mathrm{~g})+\mathrm{b} / 2 \mathrm{H}_{2} \mathrm{O}(\mathrm{I})+\mathrm{d} / 2 \mathrm{~N}_{2}(\mathrm{~g})
\end{aligned}
$$

0 requisito de definição rigorosa do estado final dos produtos de combustão impõe que os compostos orgânicos contendo enxofre ou halogéneos não possam ser estudados por SBC. Para estes últimos compostos, durante a combustão, formam-se, simultaneamente, em proporções variadas diferentes produtos de combustão, o que leva a que a técnica SBC não permita a caracterização qualitativa e quantitativa dos estados finais. É, então, usada a técnica RBC, em que se faz, imediatamente após a reacção, a rotação do vaso calorimétrico no qual se colocou uma solução apropriada, de modo a permitir uniformização do conteúdo do vaso reaccional, com a consequente possibilidade de análise de produtos. Por exemplo, na combustão de um composto orgânico clorado, grande parte dos átomos de halogéneo presentes no composto originam a formação de halogéneo molecular (15 a $20 \%$ ), segundo o descrito pela equação química (9), enquanto os outros átomos de cloro originam directamente $\mathrm{HCl}$, de acordo com a equação química gera (10).

$$
\begin{aligned}
& \mathrm{C}_{\mathrm{a}} \mathrm{H}_{\mathrm{b}} \mathrm{N}_{\mathrm{c}} \mathrm{Cl}_{\mathrm{d}}(\mathrm{cr}, \mathrm{I})+(\mathrm{a}+\mathrm{b}) \mathrm{O}_{2}(\mathrm{~g}) \rightarrow \\
& \rightarrow \text { a } \mathrm{CO}_{2}(\mathrm{~g})+\mathrm{c} / 2 \mathrm{~N}_{2}(\mathrm{~g})+ \\
& \mathrm{d} / 2 \mathrm{Cl}_{2}(\mathrm{~g})+\mathrm{b} / 2 \mathrm{H}_{2} \mathrm{O}(\mathrm{I}) \\
& +[\mathrm{dn}-(\mathrm{b}-\mathrm{d}) / 2] \mathrm{H}_{2} \mathrm{O}(\mathrm{I}) \rightarrow \\
& \rightarrow \mathrm{a} \mathrm{CO}(\mathrm{g})+\mathrm{c} / 2 \mathrm{~N}_{2}(\mathrm{~g})+\mathrm{d}\left(\mathrm{HCl} \cdot \mathrm{nH}_{2} \mathrm{O}\right)(\mathrm{l})
\end{aligned}
$$


Estudo tipico de um complexo cristalino por RSC

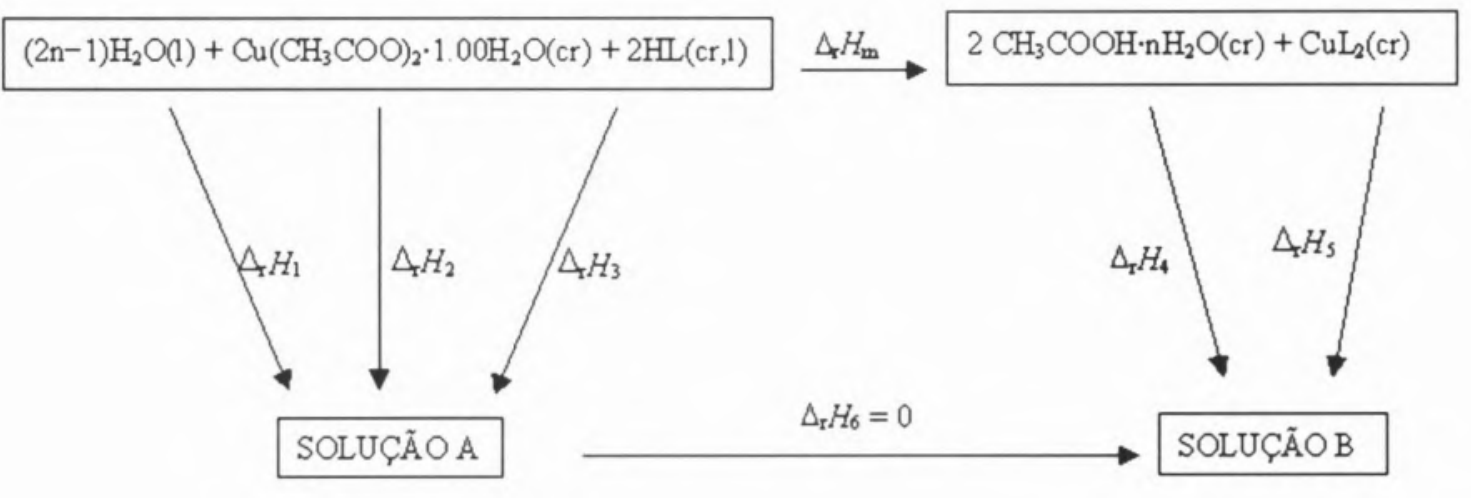

$$
\begin{gathered}
\Delta H_{\mathrm{m}}=(2 \mathrm{n}-1) \cdot \Delta_{\mathrm{r}} H_{1}+\Delta_{\mathrm{r}} H_{2}+2 \cdot \Delta_{H_{3}}-2 \cdot \Delta_{\mathrm{r}} H_{4}-\Delta_{\mathrm{r}} H_{\mathrm{s}} \\
\Delta_{\mathrm{f}} H_{\mathrm{m}}^{\circ}\left(\mathrm{CuL}_{2}, \mathrm{cr}\right)=\Delta_{\mathrm{r}} H_{\mathrm{m}}+\Delta_{\mathrm{f}} H_{\mathrm{m}}^{\circ}\left[\mathrm{Cu}\left(\mathrm{CH}_{3} \mathrm{COO}\right)_{2} \cdot 1 \cdot 00 \mathrm{H}_{2} \mathrm{O}, \mathrm{cr}\right]+2 \Delta_{\mathrm{f}} H_{\mathrm{m}}^{\circ}(\mathrm{HL}, \mathrm{cr}, 1)- \\
-\Delta_{\mathrm{f}} H_{\mathrm{m}}^{\circ}\left(\mathrm{H}_{2} \mathrm{O}, 1\right)-2 \cdot \Delta_{\mathrm{f}} H_{\mathrm{m}}^{\circ}\left(\mathrm{CH}_{3} \mathrm{COOH} e \mathrm{mnH}_{2} \mathrm{O}\right)
\end{gathered}
$$

figura 2 -- Exemplo de um esquema reaccional para o estudo de um complexo de cobre(II) com o ligando HL, a uma temperatura de referência T

Contudo, colocando inicialmente no interior da bomba de combustão uma solução de óxido de arsénio (III), aquando da rotação da bomba, o cloro molecular é reduzido a cloreto de hidrogénio, de acordo com a equação (11), pelo que a solução aquosa de cloreto de hidrogénio é o único produto clorado da reacção global descrita pela equação (10).

$$
\begin{gathered}
\mathrm{d} / 2 \mathrm{Cl}_{2}(\mathrm{~g})+\mathrm{d} / 4 \mathrm{As}_{2} \mathrm{O}_{3}(\mathrm{aq})+\mathrm{d} / 2 \mathrm{H}_{2} \mathrm{O}(\mathrm{I}) \\
\rightarrow \mathrm{d} / 4 \mathrm{As}_{2} \mathrm{O}_{5}(\mathrm{aq})+\mathrm{d} \mathrm{HCl}(\mathrm{aq})
\end{gathered}
$$

\section{Entalpias de transição de fase}

A determinação experimental directa de entalpias de sublimação, usando a técnica de microcalorimetria Calvet, ${ }^{[16]}$ tem sido efectuada usando um calorímetro diferencial de fluxo com células gémeas. Os suportes das células estão ligados a um bloco isotérmico através de um conjunto de termopares (platina/platina-ródio) todos iguais, regularmente distribuídos em torno das células e ligados em série, formando termopilhas. A diferença de potencial gerada entre as termopilhas da célula de trabalho e a de referência, dispostas simetricamente no interior do bloco calorimétrico, é proporcional à diferença de fluxo de calor das células para o referido bloco. 0 princípio de utilização do método calorimétrico para a medição de entalpias de transição de fases é indicado na figura 3.

O método de efusão de Knudsen permite a determinação indirecta de entalpias de sublimação ${ }^{[17]}$. Nesta técnica, a amostra é colocada numa célula, mantida a temperatura constante, em cuja tampa existe um orifício de dimensões muito reduzidas, através do qual pode passar um fluxo de vapor do composto, sem que o equlíbrio sólido-vapor seja perturbado. Esse fluxo é medido e relacionado com a pressão do vapor em equilibrio com a fase condensada, a uma dada temperatura, sendo a entalpia de sublimação do composto, no intervalo de temperaturas considerado, obtido pela variação das pressões de vapor com a temperatura, através da equação de Clausius-Clapeyron.

Entalpias de sublimação podem ainda ser determinadas pelo método $\mathrm{KE}$, sem conhecimento das pressões de vapor a diferentes temperaturas, desde que seja possível a medição de uma propriedade física relacionada com a taxa de efu- são $0^{[18]}$. Uma dessas propriedades é a variação da frequência de vibração de um cristal de quartzo por deposição, a baixas pressões, de composto proveniente de efusão através da célula de Knudsen.

\section{Análise de resultados}

A relação entre energética e estrutura molecular em complexos metálicos pode ser evidenciada com base em exemplos de estudos de compostos com ligações metal-oxigénio, metalazoto e metal-enxofre, designadamente complexos metálicos de aminoácidos, ${ }^{[8,9]}$ de bases de Schiff ${ }^{[14]}$ e de acilcalcogenoureias ${ }^{[12,13]}$. Em geral, as energias de dissociação metal-ligando e as correspondentes energias de dissociação hidrogénio-ligando seguem tendências paralelas, o que não constituirá uma surpresa uma vez que a energética de uma dada unidade estrutural (radical ligando) se deve reflectir na energética da molécula global em que essa unidade se insere (complexo). Em complexos metálicos do tipo $\mathrm{ML}_{\mathrm{n}}$, tem-se verificado constância dos valores da grandeza $<D H>(\mathrm{M}-\mathrm{L})-D H(\mathrm{H}-\mathrm{L})$ para um tipo par- 
ticular de ligando, $\mathrm{HL}$, o que permite inferir alguns factos:

a) Efeitos influenciando $\mathrm{DH}(\mathrm{H}-\mathrm{L})$ parecem ser compensados em $\langle D H\rangle(\mathrm{M}$ L).

b) Para um dado metal, a medição da entalpia de formação, no estado gasoso, de um qualquer complexo será suficiente para estimar os valores das entalpias de formação de outros complexos desse metal com diferentes ligandos do mesmo tipo.

c) Parece razoável, para um dado tipo de ligandos, assumir a constância de $D H(H-L)$ para comparar valores de $<D H>(M-L)$; comparações semelhantes não podem ser efectuadas quando estão envolvidos ligandos de diferentes tipos.

estudos de complexos de um dado metal com diversos ligandos do mesmo tipo. A equação (7), que permite o cálculo de $\langle D H\rangle(\mathrm{M}-\mathrm{L})-\mathrm{DH}(\mathrm{H}-\mathrm{L})$, pode ser rearranjada para incluir parâmetros na fase cristalina, obtendo-se a equação (12), válida para complexos do tipo $\mathrm{ML}_{2}$.

Para uma série de complexos de um mesmo metal, a equação (12) mostra que a representação gráfica dos valores das entalpias molares de formação de complexos, na fase cristalina, em função de idêntico parâmetro dos respectivos ligandos pode ser uma relação linear, de declive igual ao número de moléculas de ligando, $\mathrm{HL}$, por átomo metálico, $\mathrm{M}$, desde que a entalpia média de troca metal-hidrogénio, $<D H>(M-L)-D H(H-L)$, e a diferença entre entalpias de sublimação,

$\Delta_{\mathrm{f}} H_{\mathrm{m}}^{0}\left(\mathrm{ML}_{2}, \mathrm{Cr}\right)=\left\{\Delta_{\mathrm{f}} H_{\mathrm{m}}^{0}(\mathrm{M}, \mathrm{g})-2 \Delta_{\mathrm{f}} H_{\mathrm{m}}^{0}(\mathrm{H}, \mathrm{g})\right\}-2\{<D H>(\mathrm{M}-\mathrm{L})-D H(\mathrm{H}-\mathrm{L})\}$

$$
+\left\{2 \Delta_{\mathrm{cr}}^{\mathrm{g}} H_{\mathrm{m}}^{0}(\mathrm{H}-\mathrm{L})-\Delta_{\mathrm{cr}}^{\mathrm{g}} H_{\mathrm{m}}^{0}\left(\mathrm{ML}_{2}\right)\right\}+2 \Delta_{\mathrm{f}} H_{\mathrm{m}}^{0}(\mathrm{HL}, \mathrm{cr})
$$

Nesta base, tem vindo a ser realizados estudos de complexos de um ligando particular com vários metais, bem como
$2 \Delta_{\mathrm{cr}}^{\mathrm{g}} H_{\mathrm{m}}^{\mathrm{O}}(\mathrm{HL})-\Delta_{\mathrm{cr}}^{\mathrm{g}} H_{\mathrm{m}}^{0}\left(\mathrm{ML}_{2}\right)$, sejam constantes para esse conjunto de complexos.
Apesar da representação gráfica da função $\Delta_{\mathrm{f}} H_{\mathrm{m}}^{\mathrm{O}}\left(\mathrm{ML}_{2}, \mathrm{Cr}\right)=f\left(\Delta_{\mathrm{f}} H_{\mathrm{m}}^{\mathrm{O}}(\mathrm{HL}, \mathrm{cr})\right)$ poder conduzir a alguma informação relativa à grandeza $<D H>(\mathrm{M}-\mathrm{L})-D H(\mathrm{H}-$ L), não permite por si só o conhecimento do seu valor. O cálculo do valor de $<D H>(\mathrm{M}-\mathrm{L})-D H(\mathrm{H}-\mathrm{L})$ exige o conhecimento das entalpias de sublimação de ligandos e complexos, conforme se infere da equação (12). Alternativamente, a representação gráfica da função $\Delta_{\mathrm{f}} H_{\mathrm{m}}^{0}$ $\left(\mathrm{ML}_{2}, \mathrm{~g}\right)=f\left(\Delta_{\mathrm{f}} H_{\mathrm{m}}^{0}(\mathrm{HL}, \mathrm{g})\right)$ permite também o cálculo de $\langle D H\rangle(\mathrm{M}-\mathrm{L})-D H(\mathrm{H}$ L), conforme se infere da equação (7), embora a escassez de resultados na fase gasosa nem sempre o permita.

O interesse de correlações e de representações deste tipo é evidente na avaliação crítica de resultados termoquímicos, como é exemplificado por alguns estudos descritos na literatura e a seguir referidos. Assim, os valores das entalpias molares de formação padrão, no estado cristalino, de alguns complexos de $\mathrm{Cu}(\mathrm{II})$ e Ni(II) com aminoácidos simples (ligandos bidentados, tendo como átomos dadores $\mathrm{N}$ e O), em função dos correspondentes valores das entalpias de formação daqueles ligandos, no esta-

\section{Método calorimétrico: Princípio de utilização da técnica $M C$ para a mediçãa de entalpias de transição de fase}

$$
\mathrm{X}(\mathrm{cr} \text { ou } 1,298 \mathrm{~K}) \rightarrow \mathrm{X}(\mathrm{g}, \mathrm{T})
$$

$$
\Delta_{\mathrm{cr}, 1}^{\mathrm{g}} H_{\mathrm{m}}^{\mathrm{o}}(298 \mathrm{~K})
$$

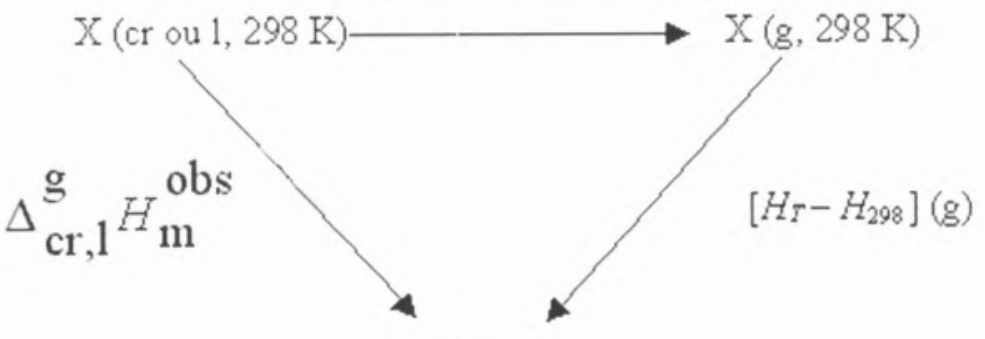

$\mathrm{X}(\mathrm{g}, T)$

$$
\Delta_{\mathrm{cr}, 1}^{\mathrm{g}} H_{\mathrm{m}}^{\mathrm{o}}(298 \mathrm{~K})=\Delta_{\mathrm{cr}, 1}^{\mathrm{g}} H_{\mathrm{m}}^{\mathrm{obs}}-\left[H_{T}-H_{298}\right](\mathrm{g})
$$




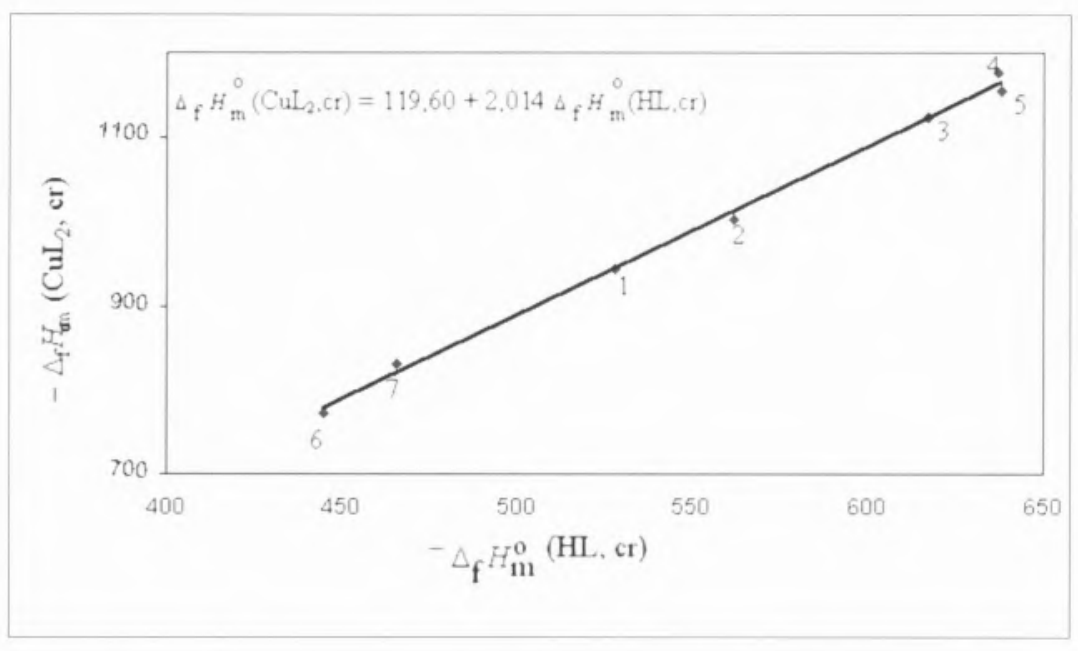

figura 4 -- Variação das entalpias de formação de complexos de Cu(II) com aminoácidos, $\mathrm{CuL}_{2}$, no estado cristalino, em função das entalpias de formação dos respectivos ligandos, HL, no estado cristalino

(HL: 1-glicina, 2-alanina, 3-valina, 4-leucina, 5-isoleucina, 6-fenilglicina, 7-fenilalanina)

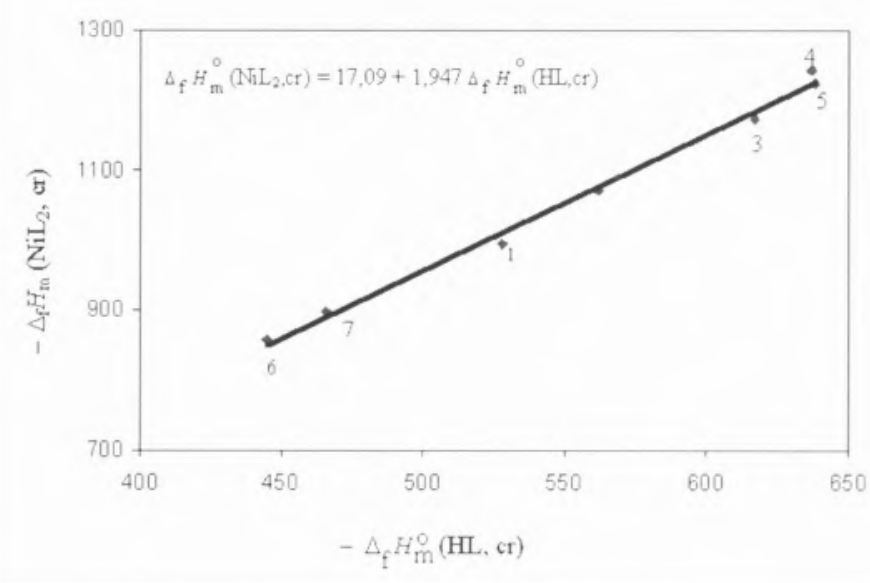

figura 5 - Variação das entalpias de formação de complexos de Ni(II) com aminoácidos, $\mathrm{NiL}_{2}$, no estado cristalino, em função das entalpias de formação dos respectivos ligandos, HL, no estado cristalino

(HL: 1-glicina, 2-alanina, 3-valina, 4-leucina, 5-isoleucina, 6-fenilglicina, 7-fenilalanina)

do cristalino, obedecem a uma relação linear com um declive de valor dois, conforme é evidenciado nos diagramas apresentados nas figuras 4 e 5 . Para cada um destes conjuntos de complexos de $\mathrm{Cu}$ (II) e Ni(II), só foi medido experimentalmente o valor da entalpia de sublimação do complexo de glicina, o que apenas permite conhecer o valor da entalpia de formação, no estado gasoso, do respectivo glicinato metálico. Para os dois glicinatos de $\mathrm{Cu}$ (II) e Ni(II), foi também possivel calcular os valores de $<D H>$ (M-glicinato) $-D H($ H-glicinato $)$, respectivamente $-19.7 \mathrm{~kJ} \mathrm{~mol}^{-1}$ e -65.8 4,4'-etilenodinitrilobis(pentano-2-ona), $\mathrm{H}_{2}$ acacen, e 1,1'-difenil-3,3'-etilenodinitrilobis(bu-tano-1-ona), $\mathrm{H}_{2}$ bzacen. Os valores de $D H(M-L)-2<D H>(H-L)$ para os complexos $\mathrm{Cu}(\text { acacen) })_{2}$, Cu(bzacen $)_{2}$, Ni(acacen) $)_{2}$ e Ni(bzacen) $)_{2}$ estão resumidos na tabela 1 , em que se apresentam, para efeitos comparativos, os resultados referentes a $\langle D H>(M-L)-$ $D H(H-L)$ em complexos do tipo $\mathrm{ML}_{2}$, com ligandos bidentados cujos átomos coordenadores são também N e O (glicina e 8-hidroxiquinolina). Os resultados da tabela 1 confirmam outros estudos em que o mesmo metal ligado a uma dada classe de ligandos, implica uma constância de valor de $D H(M-L)$ $2<D H>(H-L)$, o que pode traduzir que, para um dado tipo de ligando, os efeitos influenciando $D H(H-L)$ são compensados em $D H(M-L)$. Contudo, não é possível fazer tais comparações quando estão envolvidas diferentes classes de ligandos, como é evidenciado na tabela 1 para os glicinatos e hidroquinolinatos metálicos. Dos valores apresentados na tabela 1 podem calcular-se o valores médios de $D H(M-L)-2<D H>(H-L)$ para os complexos de $\mathrm{Cu}(\mathrm{II})$ e $\mathrm{Ni}(\mathrm{II})$ com as duas bases de Schiff, que correspondem a $(-202.9 \pm 7.1) \mathrm{kJ} \mathrm{mol}^{-1}$ e $(-21.4$ $\pm 7.1) \mathrm{kJ} \mathrm{mol}^{-1}$, respectivamente. A diferença entre estes valores médios para compostos de $\mathrm{Cu}(\mathrm{II})$ e $\mathrm{Ni}(\mathrm{II}) \mathrm{com}$ as referidas bases de Schiff, $(182 \pm 10) \mathrm{kJ}$ $\mathrm{mol}^{-1}$, pode ser comparada com as diferenças entre os valores de $2<D H>(M-L)$ $-2 D H(H-L)$ para os glicinatos e hidroxiquinolinatos de $\mathrm{Cu}(\mathrm{II})$ e $\mathrm{Ni}(\mathrm{II})$, respectivamente $(171 \pm 15)$ e $(144 \pm 14) \mathrm{kJ}$ $\mathrm{mol}^{-1}$, um valor pouco inferior mas com a mesma tendência. Os resultados apontam, ainda, para o facto de os quinolinatos e os glicinatos metálicos, envolvendo anéis de 5 lados, se ligarem de forma mais forte do que as bases de Schiff, cujos complexos apresentam anéis de 6 lados; por outro lado, a "ponte" etileno força os complexos de bases de Schiff a adoptar uma configuração planar cis, que pode ser distorcida para uma forma tetraédrica.

O estudo da energética da formação de ligações metal-ligando em quelatos metálicos tem sido alargada também a complexos metálicos de acilcalcogenou- 
reias, RCONHCXNR ${ }_{2}^{\prime}$ ( $R=$ grupo alquilo ou fenilo; $R^{\prime}=$ substituinte alquilo; $\mathrm{X}=\mathrm{O}$, S), contendo conjuntos $\mathrm{O}_{4}$ ou $\mathrm{O}_{2} \mathrm{~S}_{2}$ como átomos coordenadores, com o objectivo de avaliar a influência da vizinhança na energética da ligação. 0 estudo do complexo $N$-benzoil- $N^{\prime}, N^{\prime}$-dietilureato de $\mathrm{Cu}(\mathrm{II}), \mathrm{Cu}\left[\mathrm{PhCONCON}(\mathrm{Et})_{2}\right]_{2}$, abreviadamente designado por $\mathrm{Cu}(\mathrm{BETU})_{2}$, conduziu ao valor $\langle\mathrm{DH}\rangle(\mathrm{M}-\mathrm{L})-D H(\mathrm{H}$ $\mathrm{L})=(-70 \pm 8) \mathrm{kJ} \mathrm{mol}^{-1}{ }^{1131}$. Estimando para o ligando, HBETU, um valor $D H(\mathrm{~N}-\mathrm{H})=(365 \pm 6) \mathrm{kJ}$ mol:[19!, o valor de $\langle D H\rangle(M-L)$ resultante para o complexo Cu(BETU) é $(295 \pm 10) \mathrm{kJ}^{\mathrm{mol}-1}$; consequentemente se se admitir que. em fase gasosa, as duas ligações cobre-oxigénio no complexo são equivalentes, a entalpia média de dissociação cobre-oxigénio para o complexo $\mathrm{Cu}(\mathrm{BETU})_{2}$ é igual a $\langle\mathrm{DH}\rangle(\mathrm{Cu}-\mathrm{O})=$ $(148 \pm 10) \mathrm{kJ}$ mol-1. A diferença entre este valor e o encontrado para $\beta$-dicetonatos de cobre ${ }^{11.21}$ não é suficiente para que, atendendo às incertezas experimentais associadas, possam ser considerados discordantes.

Diversos aciltioureatos metálicos, M(RCONCSNR $\left.{ }_{2}^{\prime}\right)_{2}$, têm também sido alvo de atenção com vista à avaliação das entalpias médias de dissociação metal-enxofre nesses complexos. Como exemplo, podem referir-se os complexos de $\mathrm{Cu}$ (II) e Ni(II) com o ligando bidentado do tipo $\mathrm{HL}, N, N$-dietil- $N$-pivaloiltiourea, ('Bu)CONHCSN(Et) $)_{2}$, representados abreviadamente por $\mathrm{Cu}(\mathrm{PVET})_{2}$ e $\mathrm{Ni}(\mathrm{PVET})_{2}$, cujos valores das entalpias médias de dissociação metal-ligando, $<D H>(\mathrm{M}-\mathrm{L})$, recentemente calculados com base em determinações experimentais, ${ }^{\mid 12]}$ sāo $(310 \pm 8) \mathrm{kJ} \mathrm{mol}^{-1} \mathrm{e}$ (390 $\pm 8) \mathrm{kJ} \mathrm{mol}^{-1}$, respectivamente. Se se admitir que os valores de $\mathrm{DH}(\mathrm{M}-\mathrm{O})$ nestes compostos são idênticos aos valores $\langle D H\rangle(\mathrm{Cu}-\mathrm{O})$ em $\mathrm{Cu}(\mathrm{BETU})_{2}$ e $<D H>(\mathrm{Ni}-\mathrm{O})$ em $\beta$-dicetonatos de $\mathrm{Ni}(\mathrm{II})$, respectivamente $(148 \pm 10) \mathrm{kJ} \mathrm{mol}^{-1113:}$ e $(173 \pm 6) \mathrm{kJ} \mathrm{mol}^{-1.11]}$ obtêm-se os valores $\left\langle D H>(C u-S)=(162 \pm 13) \mathrm{kJ} \mathrm{mol}^{-1}\right.$ para $\mathrm{Cu}(\mathrm{PVET})_{2} \mathrm{e}\langle\mathrm{DH}\rangle(\mathrm{Ni}-\mathrm{S})=(217 \pm$ 18) $\mathrm{kJ} \mathrm{mol}^{-1}$ para Ni(PVET). Os resultados parecem apontar para um menor valor de entalpias de dissociação metalenxofre nos aciltioureatos do que nos monotio-ß-dicetonatos, ${ }^{(11)}$ contrastando

tabela 1 Comparação de valores $\mathrm{DH}(\mathrm{M}-\mathrm{L})-2<\mathrm{DH}>(\mathrm{H}-\mathrm{L})$ em complexos tipo $\mathrm{ML}$ com $<\mathrm{DH}>(\mathrm{M}-\mathrm{L})-$ $\mathrm{DH}(\mathrm{H}-\mathrm{L})$ em complexos tipo $\mathrm{ML}_{2}$

\begin{tabular}{|c|c|c|}
\hline Complexo & $\begin{array}{c}D H(\mathrm{M}-\mathrm{L})-2<D H>(\mathrm{H}-\mathrm{L}) / \\
/ \mathrm{kJ} \mathrm{mol}^{-1[14]}\end{array}$ & $\begin{array}{c}2<D H>(\mathrm{M}-\mathrm{L})-2 D H(\mathrm{H}-\mathrm{L}) / \\
/ \mathrm{kJ} \mathrm{mol}^{-1}\end{array}$ \\
\hline [Cu(acacen)] & $-200.6 \pm 4.7$ & \\
\hline [Cu(bzacen)] & $-205.1 \pm 9.5$ & \\
\hline [Cu(quin) ${ }_{2}$ ] & & $-150 \pm 8^{[10]}$ \\
\hline$\left[\mathrm{Cu}(\mathrm{glyO})_{2}\right]$ & & $-39 \pm 10^{[8]}$ \\
\hline [Ni(acacen)] & $-22.5 \pm 4.7$ & \\
\hline [Ni(bzacen)] & $-20.3 \pm 9.4$ & \\
\hline [Ni(quin) ${ }_{2}$ ] & & $-6 \pm 10^{1101}$ \\
\hline$\left[\mathrm{Ni}(\mathrm{glyO})_{2}\right]$ & & $132 \pm 10^{\mid 9]}$ \\
\hline
\end{tabular}

Hquin = 8. hidroxiquinolina; GlyOH = glicina

tal tendência com a tendência inversa para a dissociação metal-oxigénio. A comparação de dados cristalográficos para um monotio- $\beta$-dicetonato de níquel(II), ${ }^{[201}$ e N,N-dietil-N-pivaloiltiureato de niquel(II), ${ }^{[12]}$ referentes a comprimentos de ligação $\mathrm{Ni}$-S e Ni-O, confirma tal tendência: no pivaloiltioureato verifica-se uma ligeira diminuição do comprimento da ligaçāo Ni-O e um aumento do comprimento da ligação Ni-S.

Esta análise de resultados termoquímicos em complexos metálicos, não sendo de modo algum exaustiva para qualquer das classes de compostos referidas, evidencia alguns aspectos interessantes do estudo energético de complexos, com a consequente contribuição para o estabelecimento de relações entre características estruturais e dados energéticos das espécies químicas estudadas. Há um trabalho considerável a desenvolver no que se refere ao estudo de complexos com ligaçōes metal-enxofre e metalazoto, embora as determinaçōes experimentais necessćrias sejam muitas vezes limitadas por características de baixa solubilidade e baixa volatilidade inerentes a esses compostos.

Agradecimentos

Agradece-se à Fundação para a Ciência e Tecnologia o financiamento da Linha 5 do Centro de Investigação em Química da Universidade do Porto.

\section{Bibliografia}

[1] M. A. V. Ribeiro da Silva, M. L. C. C. H. Ferrão, Pure \& Appl. Chem., 60, 1225 (1988).

[2] M. A. V. Ribeiro da Silva, J. M. Gonçalves, J. Chem. Thermodynamics., $\mathbf{3 0}$, 1465 (1998).

[3] M. A. V. Ribeiro da Silva, M. D. M. C. Ribeiro da Silva, M. C. S. S. Rangel, G. Pilcher, M. J. Akello, Thermochimica Acta., 160, 2677 (1990).

[4] M. A. V. Ribeiro da Silva, A. M. M. V. Reis, J. Chem. Thermodynamics, 21,167 (1989).

[5] M. A. V. Ribeiro da Silva, A. M. M. V. Reis, J. Chem. Thermodynamics. 21,423 (1989)

[6] M. A. V. Ribeiro da Silva, A. M. M. V. Reis, J. Chem. Thermodynamics, 24,401 (1992)

[7] M. A. V. Ribeiro da Silva, A. M. M. V Reis, R. I. M: C. P. Faria, J. Chem. Thermodynamics, 27,1365 (1995) .

[8] M.A.V. Ribeiro da Silva, M. D. M.C Ribeiro da Silva, M. M. C. Bernardo, L. M. N. B. F. Santos, Thermochimica Acta, 205, 99 (1992). 
[9] M.A.V. Ribeiro da Silva; M. D. M.C. Ribeiro da Silva; J. A. B. A. Tuna; L. M. N. B. F. Santos, Thermochimica Acta, 205, 115 (1992).

[10] M.A.V. Ribeiro da Silva, M. A. R. de Matos, J. M. Gonçalves, Thermochimica Acta, 247, 245 (1994).

[11] L. M. N. B. F. Santos, Dissertação de Doutoramento, Faculdade de Ciências, Universidade do Porto (1995).

[12] L. C. M. da Silva, Dissertação de Doutoramento, Faculdade de Ciências, Universidade do Porto (2000).

[13] M. A. V. Ribeiro da Silva, M. D. M. C. Ribeiro da Silva, L. C. M. da Silva, F. Dietze, E. Hoyer, Thermochimica Acta, 378, 45 (2001).
[14] M. A. V. Ribeiro da Silva, M. D. M. C. Ribeiro da Silva, M. J. S. Monte, J. M. Gonçalves, E. M. R. Fernandes, J. Chem. Soc. Dalton, 1257 (1997).

[15] J. A. Martinho Simões, M. A. V. Ribeiro da Silva, em "Energetics of Stable Molecules and Reactive Intermediates" (M. E. Minas da Piedade, Ed.), NATO-

ASI Series n C535, Kluwer, Dordrecht (1999).

[16] F. A. Adedeji; D. L. S. Brown, J. A. Connor, M. Leung, M. I. Paz Andrade, H.A. Skinner, J. Organometallic Chem., 97, 221 (1975).

[17] M.A.V. Ribeiro da Silva, M. J. S. Monte, Thermochimica Acta, 171, 169 (1990).
[18] J. M. Gonçalves, Dissertação de Doutoramento, Faculdade de Ciências, Universidade do Porto (1996).

[19] P. A. MacFaul, D. D. M. Wayner, K. U. Ingold, . J. Org. Chem., 62, 3413 (1997).

[20] J. Coetzer, J. C. A. Boeyens, J. Cryst. Mol. Struct., 1, 277 (1971).

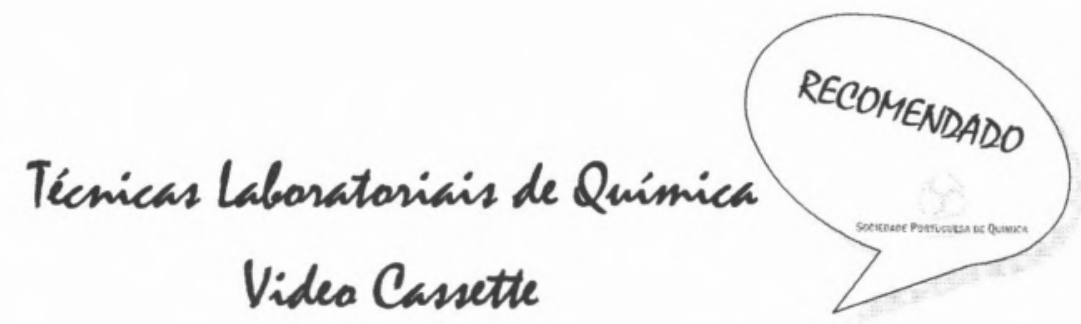

Com 7 blocos curtos e independentes, este trabalho foi concebido para apoiar as aulas de Técnicas Laboratoriais de Química destinadas a alunos do Ensino Secundário e das cadeiras introdutórias de Química do Ensino Superior. Os procedimentos apresentados são clássicos, simples e adequados para estes níveis de ensino, onde a transparência dos princípios químicos a ilustrar e a necessidade de adopção de boas práticas laboratoriais são da maior importância formativa.

Índice
Pesagem e Preparação de Soluç̃̃es (11 minutos)
Operação de balanças técnicas e de precisão
Preparação de soluções rigorosas enão rigorosas
Análise Volumétrica Quantitativa (II mimutos)
Operação com pipetas e buretas
Titulações manuais
Recristalização e Filtração (24 minutos)
Recristalização por dissolução e arrefecimento
Filtração em papel e à trompa
Filtração a quente
ExtraçãaLiquido -Liquido (7 minutos)
Operação com ampolas de decantação
Destilação (23 minutos)
Destilações simples, fraccionada,
a pressão reduzida e por arrastamento de vapor
TLC e Pontos de Fusão (9 minutos)
Cromatografia de Camada Fina
Enchimento de capilares para p.f.
Sopragem de Vidro (11 mimutos)
Estirar tubos capilares
Cortar e dobrar tubos de vidro
Demonstração do fabrico e reparação de material de
vidro executada por sopradores de vidro profissionais do IST.

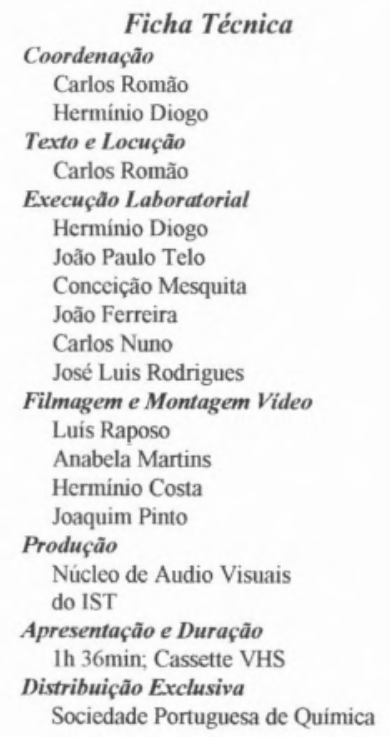

Encomendas à SPQ, Av. da República 37, 4 1050 Lisboa

Tel: 217934637 / Fax: 217952349

Preço: Instituições e não sócios $7500 \$ 00+$ IVA + portes Sócios $6000 \$ 00+$ IVA + portes 